\title{
REMARK ON CONTINUOUS COLLECTIONS
}

\author{
DEANE MONTGOMERY
}

\begin{abstract}
The result generalizes an earlier theorem of the author which showed that pointwise periodic homeomorphisms of a connected manifold are periodic.
\end{abstract}

Newman's work on orbits of periodic transformations [4] has been extended to continuous collections of finite sets by Černavskii [1], (see also [2]). The extension does not appear to apply directly to orbits of pointwise periodic transformations [3]. It is shown here that the assumption of continuity in [1] can be replaced by a slightly weaker assumption which implies continuity, and then orbits of pointwise periodic transformations become included. These results are for manifolds.

Denote by $H$ a collection of nonoverlapping finite sets filling a connected manifold $M$. All considerations here reduce to the case where $M$ has no boundary and this is assumed. Let $H(x)$ be the finite set of the collection which includes $x$, and let $H(U)$ be the union of all $H(x), x \in U$. If $H(U)=U, U$ is called saturated. The number of points in $H(x)$ is called the multiplicity of $x$ or $H(x)$ and is denoted by $\alpha(x)$.

If $H$ is continuous, [1] shows it has bounded multiplicity and also that points of maximum multiplicity are everywhere dense. These facts are used here to give the same conclusion with a slightly weaker hypothesis. Using [1] the argument is analogous to [3] where the basis is [4].

THEOREM. Let $H$ be a collection of nonoverlapping finite sets filling a connected manifold $M$. Then $H$ is continuous and, hence, of bounded multiplicity if it satisfies

(a) $H$ is lower semicontinuous,

(b) $H$ is continuous on a satuated subset if $\alpha(x)$ is bounded on the subset.

Proof. The function $\alpha(x)$ is lower semicontinuous by (a) and so its points of continuity are everywhere dense. They form an open set since $\alpha$ has integer values. Let $K$ be the closed set where the least upper bound of $\alpha$ is infinite. Then $\alpha$ is locally bounded on $M-K$, and, by (b), $H$ is continuous on $M-K$.

There is a decomposition space $X^{*}$ of $M-K$ and a proper, open, and closed map $f: M-K \rightarrow X^{*}$. Notice that $X^{*}$ is locally connected because $M-K$ is locally connected. The following lemma is known and can also be verified by the reader.

Received by the editors February 22, 1982.

1980 Mathematics Subject Classification. Primary 57S99, 57S19.

Key words and phrases. Finite-to-one mappings, continuous collections, pointwise periodic homeomorphisms.

(C)1983 American Mathematical Society $0002-9939 / 82 / 0000-1180 / \$ 02.00$ 
Lemma. Let $U^{*}$ be an open connected set in $X^{*}$. Then $f^{-1}\left(U^{*}\right)$ is open and each component maps onto $U^{*}$, that is, each component of $f^{-1}\left(U^{*}\right)$ intersects $f^{-1}\left(x^{*}\right)$ for all $x^{*} \in U^{*}$. The number of such components is finite.

To check the lemma, observe that if $V$ is a component of $f^{-1}\left(U^{*}\right)$, then $f(V)=V^{*}$ is an open connected set in $U^{*}$. If $V^{*} \neq U^{*}$, let $x^{*}$ be a point of $U^{*}$ in the boundary of $V^{*}$. Choose $x_{i}^{*} \in V^{*}$ where $x_{i}^{*} \rightarrow x^{*}$. Then choose $x_{i} \in f_{-1}\left(x_{i}^{*}\right)$ so that $x_{i} \rightarrow x$, $x_{i} \in V$. But then $x \in f^{-1}\left(U^{*}\right)$ and, hence, $x \in V$ because $V \cup\{x\}$ is connected. The rest of the lemma follows.

To proceed with the proof of the Theorem, let $U$ be a component of $M-K$. Define a collection $H^{\prime}$ in $U$ by $H^{\prime}(x)=H(x) \cap U$, for $x \in U$. Then $H^{\prime}$ is continuous on $U$ by (b). Since $U$ is a manifold, it follows from Theorem 1 of [1] that $H^{\prime}$ is bounded on $U$. A similar fact is true for each component of $H(U)$. Because $H(U)$ has only a finite number of components, $H$ is bounded on $H(U)$.

The set $K$ is nowhere dense. The function $\alpha \mid K$, like $\alpha$, is locally constant (on $K$ ) at a point of continuity. If $U$ is the only component of $M-K$, then $H^{\prime}(x)=H(x)$ and both are bounded in $U$. If $K$ is nonnull, let $p$ be a point of $K$ where $\alpha \mid K$ is continuous. Then $\alpha(x)$ is bounded in a neighborhood of $p$ by the bound of $\alpha(x)$ in $U$. Hence $K$ is null, $U=M$, and the Theorem is true.

Assume now the Theorem is false so that $K$ must separate $M$. Let $p$ be a point of $K$ where $K$ separates $M$ locally and where $(\alpha \mid K)(x)$ is continuous.

Let $p_{1}=p, p_{2}, \ldots, p_{\alpha(p)}$ be the points of $H(p)$. At each $p_{i}, K$ separates locally because $K$ has locally homeomorphic neighborhoods (in $K$ ) at these points. Let $V_{i}$, $i=1, \ldots, \alpha(p)$, be open connected sets in $M$ satisfying:

(1) $p_{i} \in V_{i}$;

(2) $V_{i}-K$ is not connected;

(3) $(\alpha \mid K)(x)=\alpha(p), x \in K \cap V_{i}$;

(4) for $x \in K \cap V_{i}, H(x) \cap V_{i}=\{x\}$;

(5) $\bar{V}_{i} \cap \bar{V}_{j}=\varnothing, i \neq j$.

Let $U$ be a component of $M-K$ which intersects $V_{1}$. An argument will now be made concerning this component $U$ which will then apply to any component of $M-K$ which intersects $V_{1}$.

Now $\bar{U} \cap\left(V_{1} \cap K\right) \neq \varnothing$. Let $q$ be a point of $\bar{U} \cap\left(V_{1} \cap K\right)$ where this set separates locally. The collection $H$ is bounded on

$$
Z=H\left[U \bigcup_{i=1}^{\alpha(p)}\left(K \cap V_{i}\right)\right]
$$

and, by (b), is continuous on $Z$. There is a continuous open map $f$ from $Z$ to its decomposition space $Z^{*}-f: Z \rightarrow Z^{*}=f(Z)$. Choose a neighborhood of $f(q)=q^{*}$ in the space $Z^{*}$ and let $Y^{*}$ be this neighborhood with $\cup_{i=1}^{\alpha(p)} f\left(K \cap V_{i}\right)$ omitted. Assume the choice made so that $f^{-1}\left(Y^{*}\right) \subset \cup V_{i}$. Choose a connected open neighborhood $V$ of $q$ with $V \subset V_{1}$ and (closure $\left.V\right) \cap H(U) \subset f^{-1}\left(Y^{*}\right)$. If closure $H(U)$ included $q$ as an inner point, then $\alpha(x)$ would be bounded at $q$, and $q$ could not be in $K$ by definition. Therefore $V$ - closure $H(U)$ contains an inner point. 
Define $W$ as follows:

$$
W=\cup\left\{\text { all components of } f^{-1}\left(Y^{*}\right) \text { which intersect } V\right\} \text {. }
$$

Then $W \cup V$ is an open connected set in $V_{1}$. On $W \cup V$ define a collection $H^{\prime \prime}$ as follows:

$$
\begin{aligned}
& \text { for } x \in W, H^{\prime \prime}(x)=H(x) \cap W \\
& \text { for } x \in V-W . \quad H^{\prime \prime}(x)=\{x\} .
\end{aligned}
$$

Lemma. The collection $H^{\prime \prime}$ is continuous.

Let $w^{\prime}$ be a point of $W$. Then $H^{\prime \prime}\left(w^{\prime}\right)=H\left(u^{\prime}\right) \cap W$. If $y$ is near $w^{\prime} H(y)$ is near $H(w)$, and then $H(y) \cap W$ is near $H(w) \cap W$ because $W$ is open. Next suppose $v \in V-W$. Then $H^{\prime \prime}(v)=\{v\}$. If $v$ is an inner point of $V-W . H^{\prime \prime}$ is trivial at all points near $v$ so $H^{\prime \prime}$ is continuous at $v$.

Next suppose $v \in V-W$ and $v$ is not an inner point of $V-W$; then $H^{\prime \prime}$ is trivial at all points of $V-W$ and so continuous at $v$ on $V-W$. Now let $w_{n}$ be a sequence of points in $W, \lim w_{n}=v$. The point $v$ is in $K \cap V_{1}$ so $H\left(w_{n}\right) \rightarrow H(v)$. Now $v$ is the only point of $H(v)$ in $V_{1}$. Hence, $H\left(w_{n}\right) \cap V_{1} \rightarrow\{v\}$ and then $H^{\prime \prime}\left(w_{n}\right) \rightarrow\{v\}$. This proves the Lemma.

Since $H^{\prime \prime}$ is trivial on $V-W$, which contains an inner point, we see that $H^{\prime \prime}$ is trivial everywhere on $W \cup V$. It follows that $x \in V \cap U$ implies $H(x) \cap W=\{x\}$, that is, $x \in V \cap U$ implies $H(x) \cap V=\{x\}$.

Let $x \in V \cap U$, and let $I$ be an arc in $V$ joining $x$ to a point $q^{1}$ in $K \cap V$ and which lies in $U$ except for $q^{1}$. The collection $H$ is continuous on $H(I)$. If $x_{i}$ is any point of $H(x)$ in $V_{i}$, there is an arc $I_{i}$ joining $x_{i}$ to $q_{i}^{1}$, where $q_{i}^{1} \in H(q)$ and where $I_{i}$ lies in $H(I) \subset f^{-1}\left(Y_{i}^{*}\right) \cup H(q)$. The existence of such an arc follows from p. 231 of Montgomery and Zippin, Topological transformation groups.

Next choose a connected open neighborhood of $q_{i}^{1}$, say $V_{i}^{1}$, with (closure $\left.V_{i}^{1}\right) \cap$ $H(U) \subset f^{-1}\left(Y^{*}\right)$, and let

$$
W_{i}=V_{i}^{1} \cup\left\{\text { all components of } f^{-1}\left(Y^{*}\right) \text { which intersect } V_{i}^{1}\right\}
$$

As before, it follows that $x_{i}$ is the only point of $H(x)$ in $W_{i}$. Thus each $q_{i}^{1} \in H\left(q^{1}\right)$ has a neighborhood which contains at most one point of $H(x)$. There are $\alpha\left(q^{1}\right)$ such neighborhoods and their union contains $H(x)$. Hence $\alpha(x) \leqslant \alpha\left(q^{1}\right)=\alpha(q)=\alpha(p)$. This is true for every component which touches $V_{1}$, so $\alpha(x)$ is bounded at $p$ and $p$ is not in $K$. This contradiction shows that $K$ does not separate and, in fact, is null. Then $H$ is bounded as the Theorem states.

The author has benefited from helpful comments by L. McAuley and E. Robinson. 


\section{REFERENCES}

1. A. V. Černavskii, Finite-to-one open mappings of manifolds, Amer. Math. Soc. Transl. (2) 100 (1971), 253-269.

2. L. McAuley and E. Robinson, On Newman's theorem for finite-to-open one mappings on manifolds, 1981. (Preprint)

3. D. Montgomery, Pointwise periodic homeomorphisms, Amer. J. Math. 59 (1937), 118-120.

4. M. H. A. Newman, A theorem on periodic transformations of spaces, Quart. J. Math. Oxford Ser. (2) (1931), 1-8.

Department of Mathematics, Institute for Advanced Study, Princeton, New Jersey 08540 